\title{
Avaliação de tratamentos endodônticos realizados por acadêmicos de Odontologia de uma instituição de ensino superior da Paraíba, Brasil
}

Evaluation of endodontic treatments performed by students at a Dentistry College in Paraíba,Brazil Evaluación de tratamientos de endodoncia realizados por estudiantes de Odontología de una institución de educación superior en Paraíba,Brasil

Fábio Victor Dias SILVA ${ }^{1}$

Jéssica Milan GOUVEIA ${ }^{2}$

Kauana da Silva ANDRADE ${ }^{1}$

Liandra Pamela de Lima SILVA ${ }^{1}$

Thaynara Cavalcante Moreira ROMÃO ${ }^{1}$

Manuela Gouvêa Campêlo dos SANTOS ${ }^{3}$ Rachel Reinaldo ARNAUD ${ }^{4}$

${ }^{1}$ Graduando(a) do Curso de Bacharelado em Odontologia pelo Centro Universitário de João Pessoa-UNIPÊ, 58053-000, João Pessoa-PB, Brasil ${ }^{2}$ Cirurgiã-dentista formada pelo Centro Universitário de João Pessoa-UNIPÊ, 58053-000, João Pessoa-PB, Brasil

${ }^{3}$ Professora Doutora do Curso de Bacharelado em Odontologia do Instituto de Ensino Superior da Paraíba-UNIESP, 58109-303, Cabedelo-PB, Brasil

${ }^{4}$ Professora Mestre do Curso de Bacharelado em Odontologia do Centro Universitário de João Pessoa-UNIPÊ, 58053-000, João Pessoa-PB, Brasil

\section{Resumo}

Introdução: A adequada obturação dos canais radiculares é considerada um fator primordial para obtenção do sucesso da terapia endodôntica, sendo também um critério importante para avaliar a qualidade do tratamento. Objetivo: avaliar radiograficamente a qualidade dos tratamentos endodônticos realizados na Clínica Escola de Odontologia do Centro Universitário de João Pessoa (UNIPÊ). Material e Método: A amostra foi composta de 123 radiografias periapicais pós-operatórias de tratamentos realizados na Clínica de Endodontia II em um período de 4 anos. Foram avaliados o perfil sociodemográfico dos pacientes atendidos e aspectos radiográficos, como conicidade, limite apical, densidade, ocorrência de erros de obturação e presença de tecido adjacente lesionado. Os dados foram agrupados em uma planilha e posteriormente foi realizada uma análise estatística descritiva. Resultados: A maior parte dos pacientes era do sexo feminino e com faixa etária de 21 a 30 anos, enquanto o grupo dentário mais frequente foi o dos incisivos centrais superiores. Radiograficamente, observou-se que $39 \%$ dos dentes apresentavam tecidos adjacentes lesionados, $92,7 \%$ tinham obturações com conicidade cônica, 90,2\% apresentavam densidade de obturação adequada e 67,5\% com limite apical adequado. Erros de obturação foram encontrados em 15,4\% dos tratamentos endodônticos. Do total dos tratamentos avaliados, $61 \%$ encontravam-se aceitáveis de acordo com os itens limite apical e densidade da obturação. Conclusão: Conclui-se que, dentre as radiografias avaliadas, a grande maioria foi aceitável. Entretanto, algumas estratégias inovadoras devem ser adotadas pelos docentes no sentido de aumentar o índice de tratamentos aceitáveis e diminuir ainda mais o índice de erros.

Descritores: Radiografia Dentária; Tratamento do Canal Radicular; Endodontia.

\section{Abstract}

Introduction: Adequate root canal filling is considered a primary factor for the success of endodontic therapy, and is also an important criterion for assessing the quality of treatment. Objective: Radiographically, to evaluate the quality of endodontic treatments performed at the School of Dentistry Clinic of Centro Universitário de João Pessoa (UNIPÊ). Material and Method: The sample consisted of 123 postoperative periapical radiographs from treatments performed at the Endodontic Clinic in a period of 4 years. The endodontic treatments were evaluated using the sociodemographic profile of the patients treated and radiographic aspects, such as taper, apical limit, density, occurrence of obturation errors and presence of adjacent tissue lesions. The data were grouped in a spreadshe et and, subsequently, a statistical analysis was performed. Results: The patient profile was predominantly female, with the age range of 21-30 years, while the most frequent dental group treated was the upper central incisors. Radiographically, it was observed that $39 \%$ of the teeth had adjacent tissues lesions, $92.7 \%$ had fillings with conical taper, $90.2 \%$ had adequate filling density and $67.5 \%$ with adequate apical limit. Obturation errors were found in $15.4 \%$ of endodontic treatments. Out of all the treatments evaluated, $61 \%$ were acceptable according to apical limit and density of obturation. Conclusion: In conclusion, among the radiographs evaluated, the great majority was acceptable. However, some innovative strategies should be adopted by instructors to increase the rate of acceptable treatments and further the rate of errors.

Descriptors: Dental Radiography; Root Canal Therapy; Endodontics.

\section{Resumen}

Introducción: El relleno adecuado del conducto radicular se considera um fator primordial para el éxito de la terapia endodôntica y también es um critério importante para evaluar la calidade del tratamento. Objetivo: evaluar radiograficamente la calidad de los tratamentos de endodoncia realizados em la Clínica Escola de Odontologia del Centro Universitário de João Pessoa (UNIPÊ). Material y Método: La muestra estuvo constituída por 123 radiografías periapicales posoperatorias de tratamentos realizados em la Clínica de Endodoncia II durante um período de 4 años. Se evaluó el perfil sociodemográfico de los pacientes atendidos y se evaluaron aspectos radiográficos, como ahusamiento, limite apical, densidade, ocurrencia de errores de obturación y presencia de tejido adyacente lesionado. Los datos se agruparon em uma hoje de cálculo y posteriormente se realizo um análisis estadístico descriptivo. Resultados: La mayoría de los pacientes eran mujeres y tenían entre 21 y 30 años, mientras que el grupo dentário más frecuente fue el de incisivos centrales superiores. Radiográficamente, se observo que el $39 \%$ de los dientes presentava lesiones em los tejidos adyacentes, el $92,7 \%$ tenía obturaciones com cono cónico, el 90,2\% tenía uma densidad de obturación adecuada y el 67,5\% com um limite apical adecuado. Se encontraron errores de obturación em el 15,4\% de los tratamentos de endodoncia. Del total de tratamentos evaluados, el $61 \%$ fueron aceptables según los ítems limite apical y densidade de llenado. Conclusión: Se concluye que, entre las radiografias evaluadas, la gran mayoría fue aceptable. Sin embargo, los profesores deben adoptar algunas estratégias inovadoras para aumentar la tasa de tratamentos aceptables y reducir aún más la tasa de errores.

Descriptores: Radiografía Dental; Tratamiento del Conducto Radicular; Endodoncia.

\section{INTRODUÇÃO}

A terapia endodôntica é constituída de várias fases, todas relacionadas entre si e com sua importância para a obtenção do sucesso no tratamento, sendo a obturação dos canais radiculares uma das fases mais importantes, já que representa o desfecho, refletindo as etapas anteriores $^{1,2}$.
A obturação endodôntica visa a restituição da função dentária normal, mediante - selamento de toda a extensão do canal radicular desde a sua porção cervical até seu término apical com homogeneidade na compactação da guta-percha, ou seja, o material obturador deve preencher todo o 
espaço outrora ocupado pelo tecido pulpar promovendo um adequado selamento em todos os sentidos a fim de impedir a entrada de bactérias. E que essa é a consagração do tratamento, pois perpetua o estado de desinfecção conseguido durante o preparo químico mecânico. Dessa forma, a obtenção do adequado selamento apical do canal radicular é considerado um fator primordial para um tratamento de qualidade e, que o sucesso do tratamento endodôntico é avaliado com base na análise radiográfica ${ }^{3-6}$.

A Associação Americana de Endodontia ${ }^{7}$, em 2019 considerou o tratamento endodôntico de qualidade, quando radiograficamente, não existam; espaços não obturados visíveis no canal, apicalmente ou lateralmente, quando houver ainda ausência de reabsorções radiculares e de rarefações ósseas. Dessa forma, pode-se dizer que a qualidade da obturação endodôntica é associada à visualização de uma imagem radiográfica do material obturador homogêneo, compacto e sem espaços e que a obturação total do espaço do sistema de canais, isolando-o do ligamento periodontal e osso alveolar assegura o sucesso no tratamento endodôntico. Portanto, busca-se a realização de obturações que permitam um preenchimento tridimensional efetivo ${ }^{8-11}$.

Vários critérios foram criados para avaliar a qualidade do tratamento do canal radicular, esses critérios baseiam-se no exame clínico, exame radiográfico ou uma combinação de ambos. Um dos métodos mais utilizados tem sido quase sempre a avaliação radiográfica. Apesar de a radiografia oferecer uma imagem bidimensional, nela pode-se observar dados importantes como a qualidade da obturação dos canais radiculares e a ausência ou não de lesão periapical $^{7}$. Assim, é recomendado que após o término do tratamento endodôntico, a qualidade do preenchimento do material obturador seja avaliada com uma radiografia ${ }^{8}$.

Neste contexto, estudos acerca da avaliação dos tratamentos endodônticos realizados por acadêmicos de odontologia tornam-se de grande relevância, haja vista que podem fornecer dados sobre a qualidade e eficácia dos tratamentos realizados em pacientes que usufruíram dos procedimentos endodônticos em clínica-escolas de odontologia, assim servindo como auxílio do progresso das pesquisas endodônticas para que possam ser oferecidos aos pacientes cada vez mais procedimentos bem sucedidos.

Dessa forma, o objetivo deste estudo foi avaliar radiograficamente a qualidade dos tratamentos endodônticos realizados por acadêmicos em um período de quatro anos na Clínica Escola de Odontologia do Centro Universitário de João Pessoa (UNIPÊ).

MATERIAL E MÉTODO

Esse estudo se trata de uma pesquisa descritiva, com abordagem quantitativa. A pesquisa foi desenvolvida nas dependências da Clínica Escola de Odontologia Professor Afonso Pereira do curso de Odontologia do UNIPÊ Centro Universitário de João Pessoa - Paraíba Brasil, abrangendo as radiografias finais de tratamentos endodônticos que integram os prontuários dos pacientes atendidos na disciplina de Endodontia II, esse que é um componente curricular do $5^{\circ}$ período do curso de Odontologia desta instituição.

- Seleção da amostra

O universo dessa pesquisa compreende os prontuários com radiografias endodônticas dos pacientes atendidos na Clínica de Endodontia do UNIPÊ no período de 4 anos consecutivos. Para a seleção da amostra foram utilizados critérios de inclusão e exclusão. Foram incluídos os prontuários que apresentassem a radiografia final pós-operatória e que esta evidenciasse qualidade adequada aos objetivos do trabalho. Foram considerados excluídos do estudo os prontuários correspondentes aos tratamentos endodônticos de molares, possíveis casos de retratamento, aqueles que apresentassem radiografias finais de difícil visualização ou que se encontrem alongadas ou com sobreposição de estruturas, inviabilizando a avaliação e radiografias finais em que o ápice radiográfico foi cortado. Por ficha clínica, foi selecionado aleatoriamente apenas um tratamento endodôntico para avaliação.

- Coleta de dados

Após a aprovação deste estudo pelo Comitê de Ética em Pesquisa (Parecer $\mathrm{n}^{\circ}$ 793.333 e CAAE: 32711714.9.0000.5176, e autorização da Coordenação da Clínica Escola de Odontologia do UNIPÊ, foi inicialmente realizada, no período de outubro a dezembro de 2014, a identificação dos prontuários e radiografias finais dos pacientes cujos tratamentos endodônticos foram realizados.

A coleta de dados foi dividida em dois momentos, inicialmente foi realizada a identificação dos prontuários cujos tratamentos endodônticos foram avaliados e, posteriormente, foi realizada a avaliação radiográfica do tratamento endodôntico das imagens obtidas, baseado no estudo de Soares, 2010.

As radiografias finais dos prontuários 
selecionados foram identificadas com um código numérico de acordo com a ordem crescente de avaliação. Posteriormente, foram digitalizadas utilizando uma Máquina Fotográfica Sony alfas Nex-c3k, sendo transferidas para um banco de dados de acesso exclusivo aos pesquisadores envolvidos no trabalho. Cada radiografia foi salva com seu código numérico e as iniciais de cada paciente evitando repetições de análises. Uma ficha de coleta de dados foi elaborada para avaliação das radiografias, contendo os critérios considerados na avaliação da qualidade dos tratamentos endodônticos realizados na Clínica Escola do UNIPÊ e os demais objetivos. Para identificação das radiografias na ficha de coleta de dados, foram utilizados o código numérico correspondente ao prontuário e radiografia final; o semestre do ano letivo acadêmico do UNIPÉ no qual foi realizado o tratamento endodôntico; o gênero do paciente, masculino ou feminino; a idade do paciente; e o número do elemento dentário que foi submetido ao tratamento endodôntico e classificado posteriormente de acordo com o grupo dentário. Em casos de elementos dentários que apresentassem mais de um canal, foi considerado o canal com pior qualidade de obturação, pois o insucesso de apenas um canal determina o insucesso de todo o tratamento endodôntico. Em casos de sobreposição de imagens de canais radiculares, a imagem visualizada foi avaliada como um único canal ${ }^{12}$.

\section{- Avaliação das radiografias}

Para a análise das radiografias foram selecionados dois examinadores especialistas em Endodontia e devidamente treinados e calibrados, que examinaram as radiografias em condições padronizadas de interpretação. Cada examinador recebeu as radiografias a serem analisadas, obtidas por meio de digitalização, junto com uma ficha de exame radiográfico contendo os critérios considerados na avaliação radiográfica em relação a qualidade das obturações realizadas e em relação aos erros encontrados. Em casos de discordância entre os pesquisadores, uma nova avaliação foi realizada para que os mesmos entrassem em consenso e definissem a dúvida existente. Em relação a qualidade das obturações realizadas, para efeitos de avaliação, foram empregados os critérios descritos no Quadro 1, adaptado de Soares ${ }^{13}$, Kamaura et al. ${ }^{14}$ e Pontes et al. ${ }^{15}$.

Em relação aos erros, foi observada a presença ou ausência dos mesmos de acordo com a seguinte classificação: excesso de gutapercha na câmara pulpar (quando o nível de corte da guta-percha exceder o colo anatômico dental; extravasamento de cimento obturador (quando apresentar extrusão de material com radiopacidade característica de cimento endodôntico; erros durante a restauração provisória (excesso, falta ou não adaptação do material restaurador).

Quadro 1 - Descrição dos critérios utilizados para avaliação radiográfica.

\begin{tabular}{|c|c|c|}
\hline CRITÉRIO & \multicolumn{1}{c|}{ CLASSIFICAÇÃo } \\
\hline Conicidade & - $\begin{array}{l}\text { Cônica (adequada, quando a obturação se } \\
\text { apresenta convergente em direção apical). } \\
\text { Paralela (inadequada, quando a obturação } \\
\text { apresentar paredes paralelas). }\end{array}$ \\
\hline Distância & - $\begin{array}{l}\text { Nível do Comprimento real do dente (CRD), } \\
\text { preenchendo completamente o canal radicular; } \\
\text { Nível de Comprimento real de trabalho (CRT), } \\
\text { abturação 1mm aquém do ápice radiográfico; } \\
\text { ápice-obturação }\end{array}$ & $\begin{array}{l}\text { Subobturado, quando se encontrava aquém de 2 } \\
\text { mm do ápice radiográfico; } \\
\text { Sobreobturado, quando a obturação } \\
\text { ultrapassava o limite o do ápice radiográfico. }\end{array}$ \\
O limite apical foi classificado como ideal \\
quando a obturação estiver localizada a 1mm aquém do \\
ápice radiográfico.
\end{tabular}

Fonte: Baseado e adaptado de Soares ${ }^{13}$, Kamaura et al. ${ }^{14}$ e Pontes et al. ${ }^{15}$.

Os tratamentos endodônticos foram considerados aceitáveis quando apresentaram os itens limite apical e densidade da obturação adequados ao mesmo tempo, ou seja, quando a imagem radiográfica mostrou adequada condensação do material obturador preenchendo toda a extensão do canal e situando-se a $1 \mathrm{~mm}$ do ápice radiográfico ${ }^{6,13}$. Porém, também foi adicionado 0 item conicidade da obturação onde foi verificado o valor percentual da quantidade de tratamentos endodônticos que possuíam os itens limite apical, densidade e conicidade ao mesmo tempo ${ }^{16}$.

Os dados coletados foram armazenados em uma planilha no programa Excel 2013 (Microsoft $(B)$, a qual agrupou e classificou todos os quesitos pertinentes a pesquisa. Posteriormente, foi realizada uma análise estatística descritiva e um tratamento estatístico percentual, por meio de dados absolutos e relativos utilizando o programa de informática SPSS Statistics para Windows, versão 20.0.

RESULTADOS

A amostra contou com 123 (64\%) fichas clínicas de um total de 192 encontradas disponíveis nos arquivos da Clínica de Endodontia II do UNIPÊ entre os períodos de 2010 a 2014. Para cada ficha clínica, apenas um tratamento endodôntico foi avaliado. A maioria dos pacientes era do gênero feminino $(65,0 \%)$. A faixa etária mais prevalente foi entre 21 e 30 anos, $24,4 \%$ do total da amostra, com 
média de idade dos pacientes atendidos de 35,07 anos, variando de 12 a 67 anos. O trabalho também evidenciou que o grupo dentário mais prevalente foi o dos Incisivos centrais superiores com $23,6 \%$ do total da amostra, seguido do segundo pré-molar superior $(20,3 \%)$, incisivo lateral superior $(14,6 \%)$ e primeiro pré-molar superior $(13,8 \%)$.

Os resultados das avaliações das obturações realizadas como vitalidade pulpar, presença de tecidos lesionados, conicidade e densidade de obturação, limite apical e erros de obturação podem ser visualizados na Tabela 1. Do total de radiografias avaliadas, 39\% apresentou tecidos adjacentes lesionados, mostrando rompimento da lâmina dura associado à área radiolúcida. Além disso, os resultados mostraram que $62,6 \%$ dos tratamentos realizados foram em polpa dentária necrosada.

Tabela 1. Distribuições absolutas e percentuais dos casos de acordo com as variáveis relacionadas à avaliação do tratamento endodôntico.

\begin{tabular}{l|c|c}
\hline \multicolumn{1}{c|}{ VARIÁVEL } & n & \% \\
\hline Polpa dentária & 46 & 37,4 \\
\hline Vital & 77 & 62,6 \\
\hline Necrosada & 48 & 39,0 \\
\hline Tecidos adjacentes lesionados & 75 & 61,0 \\
\hline Sim & 114 & 92,7 \\
\hline Não & 9 & 7,3 \\
\hline Conicidade da obturação & 111 & 90,2 \\
\hline Adequada (Cônica) & 12 & 9,8 \\
\hline Inadequada (Paralela) & 22 & 17,9 \\
\hline Densidade da obturação & 83 & 67,5 \\
\hline Adequada & 14 & 11,4 \\
\hline Inadequada & 4 & 3,3 \\
\hline Limite apical da obturação & 104 & 84,6 \\
\hline CRD & 16 & 13,0 \\
\hline CRT & 2 & 1,6 \\
\hline Aquém do limite (subobturados) & 1 & 0,8 \\
\hline Além do limite (sobreobturados) & $\mathbf{1 2 3}$ & $\mathbf{1 0 0 , 0}$ \\
\hline Erros de obturação & \multicolumn{2}{|c}{} \\
\hline Ausente & \multicolumn{1}{|c}{} \\
\hline Excesso de guta-percha na câmara pulpar & Total &
\end{tabular}

A Tabela 2 apresenta os resultados de conicidade e densidade da obturação distribuídos de acordo com o grupo dentário. Todos os dentes do grupo dos caninos superiores, incisivos inferiores e pré-molares inferiores apresentaram conicidade de obturação adequada, enquanto 0 maior percentual de conicidade paralela foi encontrado no grupo de caninos inferiores $(33,3 \%)$. Com relação à densidade da obturação, os grupos que apresentaram maior percentual de densidade adequada foram os caninos superiores $(100 \%)$, incisivos centrais inferiores $(100 \%)$ e os primeiros pré-molares inferiores $(100 \%)$. Já o maior percentual de densidade inadequada foi observado nos Incisivos laterais inferiores $(33,3 \%)$ e caninos inferiores $(33,3 \%)$.

A distribuição do limite apical de obturação de acordo com o grupo dentário pode ser observada na Tabela 3 , onde se observa que os grupos que apresentaram o maior percentual de limite adequado de obturação foram os Incisivos laterais inferiores (100\%) e caninos superiores (100\%). Além disso, foi encontrado que o grupo dos Incisivos laterais superiores e segundos pré-molares apresentaram o menor percentual de limite apical de obturação adequado (44,4\%).

Tabela 2. Distribuição absoluta e percentual dos casos de acordo com o grupo dentário, segundo a avaliação da conicidade e densidade da obturação.

\begin{tabular}{|c|c|c|c|c|}
\hline \multirow{2}{*}{$\begin{array}{c}\text { Grupo } \\
\text { Dentário }\end{array}$} & \multicolumn{2}{|c|}{$\begin{array}{c}\text { Conicidade da } \\
\text { obturação }\end{array}$} & \multicolumn{2}{|c|}{ Densidade da obturação } \\
\hline & $\begin{array}{c}\text { Cônica } \\
\text { n (\%) }\end{array}$ & $\begin{array}{c}\text { Paralela } \\
\text { n (\%) }\end{array}$ & $\begin{array}{c}\text { Adequada } \\
\text { n (\%) }\end{array}$ & $\begin{array}{c}\text { Inadequada } \\
\text { n (\%) }\end{array}$ \\
\hline ICS & $28(96,6)$ & $1(3,4)$ & $25(86,2)$ & $4(13,8)$ \\
\hline ILS & $16(88,9)$ & $2(11,1)$ & $17(94,4)$ & $1(5,6)$ \\
\hline $\mathrm{CS}$ & $10(100,0)$ & $\mathrm{o}(\mathrm{o}, \mathrm{O})$ & $10(100,0)$ & $\mathrm{o}(\mathrm{o}, \mathrm{O})$ \\
\hline $1^{\circ} \mathrm{PMS}$ & $15(88,2)$ & $2(11,8)$ & $16(94,1)$ & $1(5,9)$ \\
\hline $2^{\circ} \mathrm{PMS}$ & $22(88,0)$ & $3(12,0)$ & $23(92,0)$ & $2(8,0)$ \\
\hline ICI & $3(100,0)$ & $\mathrm{O}(\mathrm{o}, \mathrm{O})$ & $3(100,0)$ & $\mathrm{o}(\mathrm{o}, \mathrm{O})$ \\
\hline ILI & $3(100,0)$ & $\mathrm{o}(\mathrm{o}, \mathrm{O})$ & $2(66,7)$ & $1(33,3)$ \\
\hline CI & $2(66,7)$ & $1(33,3)$ & $2(66,7)$ & $1(33,3)$ \\
\hline $1^{\circ} \mathrm{PMI}$ & $6(100,0)$ & $\mathrm{o}(\mathrm{O}, \mathrm{O})$ & $6(100,0)$ & $\mathrm{o}(\mathrm{O}, \mathrm{O})$ \\
\hline $2^{\circ} \mathrm{PMI}$ & $9(100,0)$ & $0(0,0)$ & $7(77,8)$ & $2(22,2)$ \\
\hline Total & $114(92,7)$ & $9(7,3)$ & $111(90,2)$ & $12(9,8)$ \\
\hline
\end{tabular}

Fonte: Dados de pesquisa.

Legenda: ICS - Incisivos centrais superiores; ILS - Incisivos laterais superiores; CS Caninos superiores; PMS - Pré-molares superiores; $\mathrm{ICl}$ - Incisivos centrais inferiores: ILI - Incisivos laterais inferiores; CI - Caninos inferiores; PMI - Pré-molares inferiores.

Tabela 3. Distribuição absoluta e percentual dos casos clínicos de acordo com o grupo dentário, segundo a avaliação do limite apical da obbturação.

\begin{tabular}{|c|c|c|c|c|}
\hline \multirow[b]{2}{*}{$\begin{array}{l}\text { Grupo } \\
\text { Dentário }\end{array}$} & \multicolumn{4}{|c|}{ Limite apical da obturação } \\
\hline & $\begin{array}{l}\text { CRD } \\
\text { n (\%) }\end{array}$ & $\begin{array}{c}\text { CRT } \\
\text { n (\%) }\end{array}$ & $\begin{array}{c}\text { Aquém } \\
\text { do limite } \\
\text { n (\%) }\end{array}$ & $\begin{array}{c}\text { Além do } \\
\text { limite } \\
\text { n (\%) }\end{array}$ \\
\hline ICS & $8(27,6)$ & $18(62,1)$ & $2(6,9)$ & $1(3,4)$ \\
\hline ILS & $8(44,4)$ & $8(44,4)$ & $1(5,6)$ & $1(5,6)$ \\
\hline $\mathrm{CS}$ & $\mathrm{O}(0,0)$ & $10(100,0)$ & $\mathrm{O}(0,0)$ & $0(0,0)$ \\
\hline $1^{\circ} \mathrm{PMS}$ & $1(5,9)$ & $13(76,5)$ & $3(17,6)$ & $0(0,0)$ \\
\hline $2^{\circ}$ PMS & $2(8,0)$ & $19(76,0)$ & $4(16,0)$ & $\mathrm{o}(0,0)$ \\
\hline ICI & $1(33,3)$ & $2(66,7)$ & $0(0,0)$ & $\mathrm{O}(0,0)$ \\
\hline ILI & $0(0,0)$ & $3(100,0)$ & $0(0,0)$ & $\mathrm{o}(0,0)$ \\
\hline CI & $0(0,0)$ & $2(66,7)$ & $1(33,3)$ & $\mathrm{o}(0,0)$ \\
\hline $1^{\circ} \mathrm{PMI}$ & $0(0,0)$ & $4(66,7)$ & $1(16,7)$ & $1(16,7)$ \\
\hline $2^{\circ} \mathrm{PMI}$ & $2(22,2)$ & $4(44,4)$ & $2(22,2)$ & $1(11,1)$ \\
\hline Total & $22(17,9)$ & $83(67,5)$ & $14(11,4)$ & $4(3,3)$ \\
\hline
\end{tabular}

Fonte: Dados de pesquisa.

Fonte: Dados de pesquisa. Legenda: ICS - Incisivos centrais superiores, LS - Incisivos laterais superiores; CS - Incisivos laterais ineriores; $\mathrm{Cl}$ - Caninos infeior; PMI - Prémolares infeiores.

Os resultados das avaliações de erros encontrados nos tratamentos endodônticos avaliados de acordo com o grupo dentário estão agrupados na Tabela 4. Os grupos dentários que apresentaram 0 maior percentual de ausência de erros foram caninos superiores e inferiores, ambos com $100 \%$. Outrossim, o grupo que apresentou o maior percentual de erros foi o $2^{\circ}$ pré-molar inferior, com $44,4 \%$. A Tabela 5 apresenta os resultados da avaliação geral do tratamento endodôntico segundo o grupo dentário levando em consideração os critérios de qualidade. Do total de tratamentos endodônticos avaliados, 61\% encontravam-se aceitáveis. O grupo dentário que apresentou a maior percentual de dentes com tratamento endodôntico aceitável foram os caninos 
superiores, enquanto o grupo dos caninos inferiores apresentou a menor percentual.

Tabela 4. Distribuição absoluta e percentual dos casos de acordo como grupo dentário, segundo a avaliação dos erros de obturação.

\begin{tabular}{|c|c|c|c|c|}
\hline \multirow[b]{2}{*}{$\begin{array}{c}\text { Grupo } \\
\text { Dentario }\end{array}$} & \multicolumn{4}{|c|}{ Erros de obturação } \\
\hline & $\begin{array}{c}\text { Ausente } \\
\text { n(\%) }\end{array}$ & $\begin{array}{l}\text { Excesso } \\
\text { de guta- } \\
\text { percha } \\
\text { câna } \\
\text { pulmara } \\
\text { pulpar } \\
\text { n (\%) }\end{array}$ & $\begin{array}{c}\text { Extravasamento } \\
\text { de cimento } \\
\text { obturador } \\
\text { n (\%) }\end{array}$ & $\begin{array}{c}\text { Erros } \\
\text { durante a } \\
\text { restauraçãó } \\
\text { provisoria } \\
\text { n (\%) }\end{array}$ \\
\hline ICS & $23(79,3)$ & $4(13,8)$ & $2(6,9)$ & $0(0,0)$ \\
\hline ILS & $16(88,9)$ & $2(11,1)$ & $0(0,0)$ & $0(0,0)$ \\
\hline $\mathrm{CS}$ & $10(100,0)$ & $0(0,0)$ & $0(0,0)$ & $0(0,0)$ \\
\hline $1^{\circ} \mathrm{PMS}$ & $16(94,1)$ & $1(5,9)$ & $0(0,0)$ & $0(0,0)$ \\
\hline $2^{\circ} \mathrm{PMS}$ & $22(88,0)$ & $3(12,0)$ & $\mathrm{o}(0,0)$ & $0(0,0)$ \\
\hline ICI & $2(66,7)$ & $1(33,3)$ & $0(0,0)$ & $\mathrm{O}(0, \mathrm{o})$ \\
\hline ICL & $2(66,7)$ & $1(33,3)$ & $0(0,0)$ & $0(0,0)$ \\
\hline $\mathrm{CI}$ & $3(100,0)$ & $0(0,0)$ & $0(0,0)$ & $0(0,0)$ \\
\hline $1^{\circ} \mathrm{PMI}$ & $5(83,3)$ & $1(16,7)$ & $0(0,0)$ & $0(0,0)$ \\
\hline $2^{\circ} \mathrm{PMI}$ & $5(55,5)$ & $3(33,3)$ & $\mathrm{O}(0,0)$ & $1(11,1)$ \\
\hline Total & $104(84,6)$ & $16(13,0)$ & $2(1,6)$ & $1(0,8)$ \\
\hline
\end{tabular}

Tabela 5. Distribuição absoluta e percentual dos casos de acordo com o grupo dentário, segundo a avaliação do tratamento endodôntico.

\begin{tabular}{c|c|c}
\hline \multirow{2}{*}{ Grupo Dentário } & \multicolumn{2}{|c}{ Tratamento Endodôntico } \\
\cline { 2 - 3 } & $\begin{array}{c}\text { Aceitável } \\
\text { n (\%) }\end{array}$ & $\begin{array}{c}\text { Inaceitável } \\
\text { n (\%) }\end{array}$ \\
\hline ICS & $15(51,7)$ & $14(48,3)$ \\
\hline ILS & $8(44,4)$ & $10(55,6)$ \\
\hline CS & $10(100,0)$ & $0(0,0)$ \\
\hline $1^{\text {PMS }}$ & $12(70,6)$ & $5(29,4)$ \\
\hline $2^{\text {o }}$ PMS & $17(68,0)$ & $8(32,0)$ \\
\hline ICI & $2(66,7)$ & $1(33,3)$ \\
\hline ILI & $2(66,7)$ & $1(33,3)$ \\
\hline CI & $1(33,3)$ & $2(66,7)$ \\
\hline $1^{\text {O PMI }}$ & $4(66,7)$ & $2(33,3)$ \\
\hline $2^{0}$ PMI & $4(44,4)$ & $5(55,6)$ \\
\hline Total & $\mathbf{7 5 ( 6 1 , 0 )}$ & $\mathbf{4 8 ( 3 9 , 0 )}$ \\
\hline
\end{tabular}

Fonte: Dados de pesquisa.

Legenda: ICS - Incisivos centrais superiores; ILS - Incisivos laterais superiores; CS Caninos superiores; PMS - Pré-molares superiores; ICI - Incisivos centrais inferiores; ILI - Incisivos laterais inferiores; $\mathrm{Cl}$ - Caninos inferiores; PMI - Pré-molares inferiores.

DISCUSSÃO

Este estudo teve como objetivo analisar as radiografias finais dos tratamentos endodônticos que integram os prontuários dos pacientes atendidos na disciplina de Endodontia II, um componente curricular do $5^{\circ}$ período do curso de Odontologia do Centro Universitário de João Pessoa - UNIPÊ. A amostra correspondeu a $64 \%$ do total, o que mostrou um número relativamente baixo de radiografias que cumprem os critérios mínimos de qualidade adequada para avaliação, o que alerta um maior rigor dos docentes em relação a adequação das radiografias finais dos tratamentos endodônticos.

A maioria dos pacientes era do sexo feminino $(65,0 \%)$ corroborando com os resultados de Occhi et al. ${ }^{17}$, Soares ${ }^{13}$, Ferreira et al. ${ }^{18}$ e Siqueira Jr et al. ${ }^{6}$ que obtiveram maior prevalência deste gênero em $75 \%, 69,9 \%$, $72,8 \%$ e $66,3 \%$, respectivamente. Nesse estudo, a faixa etária mais prevalente foi entre 21 e 30 anos, $24,4 \%$ do total da amostra, com média de idade dos pacientes atendidos de
35,07 anos, variando de 12 a 67 anos. Esses dados foram semelhantes ao estudo de Soare ${ }^{13}$ com $26,4 \%$ de prevalência na mesma faixa de idade. Os resultados sugerem um maior cuidado do sexo feminino e dos adultos jovens com a saúde bucal, no sentido de preservar os elementos dentários na cavidade oral.

Esse trabalho evidenciou que o grupo dentário mais prevalente foi 0 dos Incisivos centrais superiores com $23,6 \%$ do total da amostra. Estes valores concordam com os de Soares ${ }^{13}$, Ferreira et al. ${ }^{18}$ e Ribeiro et al. ${ }^{19}$ que obtiveram $25,8 \%, \quad 49 \%$ e $49,6 \%$ respectivamente, de prevalência por tal grupo dentário. Entretanto, diferem dos resultados encontrados por Siqueira $\mathrm{Jr}$ et al. ${ }^{6}$, onde a maior prevalência foi de pré-molares superiores $(20,6 \%)$, e dos resultados de Zoti e Hartmann ${ }^{2}$ e Eskandarloo et al. $^{3}$, que encontraram a maior prevalência no grupo dos pré-molares em $37,8 \%$ e $45,1 \%$ dos casos analisados, respectivamente. Os percentuais observados neste estudo sugerem uma maior preocupação dos pacientes atendidos com o fator estético, já que os dentes centrais superiores foram os mais prevalentes.

Do total de radiografias avaliadas, 39\% apresentou tecidos adjacentes lesionados, mostrando rompimento da lâmina dura associado à área radiolúcida, corroborando com porcentagens relativamente semelhantes encontrados nos estudos de Pontes et al. ${ }^{15}$, Gaspar et al. ${ }^{20}$, Freitas et al. ${ }^{21}$, Silveira et al. ${ }^{22}$ e Ribeiro et al. ${ }^{19}$ com achados de $21 \%, 37 \%$, $35,5 \%, 27 \%$ e $50 \%$ respectivamente. Além disso, os resultados mostraram que dos 77 casos de polpa dentária necrosada, $62,3 \%$ apresentavam tecidos adjacentes lesionados radiograficamente, discordando do valor encontrado por Giust et al. ${ }^{23} \mathrm{com} 25 \%$.

O presente estudo obteve $92,7 \%$ dos tratamentos endodônticos com obturações cônicas, confirmando os resultados encontrados por Soares ${ }^{13}$, Kamaura et al. ${ }^{14}$ e Fong et al. ${ }^{4}$ com $97,7 \%$, $88 \%$ e $97 \%$ respectivamente. Embora os resultados tenham sido satisfatórios, devemos lembrar que foi avaliada apenas a radiografia periapical final do tratamento, um método radiográfico que contem limitações por se tratar de imagem bidimensional. Dessa forma, pode-se justificar a discordância de porcentagem do resultado encontrado no estudo de $\mathrm{Er}$ et al. ${ }^{24}$ que obtiveram frequência de conicidade adequada de $68,3 \%$ ao avaliarem mais de uma radiografia dos tratamentos endodônticos. Resultado divergente também foi encontrado por Eskandarloo et al. $^{3}$ com 44,9\% dos casos apresentando conicidade adequada. 
Ao avaliar a literatura, observam-se algumas discordâncias entre os autores em relação ao limite apical adequado. Alguns dos autores pesquisados, considera o limite mais adequado entre 0 e $2 \mathrm{~mm}$ aquém do ápice radiográfico ${ }^{3,14}$. Enquanto outros autores como Siqueira $\mathrm{Jr}$ et al. $^{6}$ consideram o limite apical adequado entre 1 e $2 \mathrm{~mm}$. $O$ presente estudo considerou como limite apical adequado o nível de comprimento real de trabalho (CRT), com a obturação $1 \mathrm{~mm}$ aquém do ápice radiográfico.

A prevalência de limite apical adequado observada nos estudos com o mesmo critério de avaliação variou de $36 \%$ a $79,8 \%$. Os resultados da avaliação do item limite apical de obturação desse estudo apresentaram valores satisfatórios, onde $67,5 \%$ encontravam-se obturados no limite adequado, corroborando com resultados observados por Occhi et al. ${ }^{17} \mathrm{de}$ $67,8 \%$. Este percentual apresenta resultado superior aos estudos de Pontes et al. ${ }^{15}$ (2013) de $48 \%$, Gaspar $\mathrm{Jr}$ et al. ${ }^{20}$ de $47 \%$ e Freitas et al. $^{21}$ de $36 \%$. Em contrapartida, apresentou resultado inferior ao de Soares ${ }^{13}$ de $79,8 \%$ e Costa et al. ${ }^{25}$ de $80 \%$. Já Eskandarloo et al. ${ }^{3}$ e Fong et al. ${ }^{4}$ encontraram resultados diferentes em seus estudos, onde foram considerados com comprimento adequado $70,8 \%$ e $72,2 \%$ dos casos analisados, respectivamente. Essa diferença pode ser justificada em razão do critério utilizado pelos autores, sendo considerado um comprimento adequado quando o final da obturação se localizava de 0,0 a 2,0 $\mathrm{mm}$ aquém do ápice radiográfico.

Dos percentuais de limite apical inadequado 0 presente estudo apresentou $11,4 \%$ dos casos subobturados e $3,3 \%$ sobreobturados, corroborando com outras pesquisas, que apresentaram uma maior prevalência de subobturações, em detrimento das sobreobturações, como as de Occhi et al. ${ }^{17}$ com $25 \%$ e $7,14 \%$, Gaspar et al. ${ }^{20}$ com $41 \%$ e $12 \%$, Costa et al. ${ }^{25}$ com $17,5 \%$ e $2,5 \%$, Eskandarloo et al. ${ }^{3}$ com $17,1 \%$ e $12,2 \%$ e Fong et al. $^{4}$ com $23,3 \%$ e $4,5 \%$, respectivamente. No entanto, esses valores vão de encontro aos obtidos no estudo de Pontes et al. $^{15}$ que revelaram $18 \%$ dos tratamentos sobreobturados e $3 \%$ subobturados.

Os resultados referentes ao item densidade de obturação mostraram que a maioria dos tratamentos avaliados foram adequados, satisfazendo um total de $90,2 \%$ dos casos analisados. Esses achados corroboram com os resultados encontrados por Fong et al. ${ }^{4}$ e Vukadinov et al. ${ }^{26}$, sendo observado uma densidade satisfatória em $90,8 \%$ e $92,6 \%$ dos casos, respectivamente. Entretanto, quando comparado com os resultados obtidos na literatura, observamos uma diferença superior aos de Soares ${ }^{13}$ com 50,8\%, Gaspar Jr et al..$^{20}$ com $54 \%$, Freitas et al. ${ }^{21}$ com $31 \%$ e de Eskandarloo et al. $^{3}$ que obteve apenas 25,2\% dos casos com densidade adequada.

Para avaliar a qualidade do tratamento endodôntico em termos gerais foram selecionados os tratamentos que obtiveram os critérios de avaliação limite apical e densidade de obturação adequados ao mesmo tempo. Do total de tratamentos endodônticos avaliados, $61 \%$ encontravam-se aceitáveis. Do mesmo modo, foram levados em consideração os três parâmetros simultaneamente, limite apical, densidade adequada e conicidade cônica, onde houve uma queda percentual para $60,2 \%$, assim como o resultado de Soares ${ }^{13}$, que houve uma queda de $40 \%$ para $39 \%$ quando 0 item conicidade foi acrescentado.

Os estudos com o mesmo critério de avaliação de qualidade de tratamento endodôntico apresentaram percentuais que variavam de $40 \%$ a $96 \%$ dos casos aceitáveis. Resultados inferiores a essa pesquisa foram encontrados por Soares ${ }^{13}$ com $40 \%$, Gaspar Jr et al. ${ }^{20} \mathrm{com} 55 \%$, Freitas et al. $^{21} \mathrm{com} 48 \%$, Eskandarloo et al. ${ }^{3}$ com $10,4 \%$ e Román-Richon et $\mathrm{al}^{27}$ com $44 \%$. Contudo, resultados superiores foram observados nos estudos de Pontes et al. ${ }^{15}$ com $81 \%$, Fong et al. ${ }^{4}$ com $66 \%$, Occhi et al. ${ }^{17}$ com $96 \%$ e Ribeiro et al. ${ }^{21}$ com $71,9 \%$. O elevado percentual observado no estudo de Occhi et al. ${ }^{17}$ pode ser explicado pelo baixo número radiografias, ou seja, uma amostra de apenas 28 tratamentos endodônticos avaliados. Alves-Silva ${ }^{28}$ avaliaram a qualidade das radiografias periapicais realizadas durante as etapas do tratamento endodôntico na clínica de graduação de um curso de Odontologia e observaram alongamento da imagem radiográfica, erros na conometria e odontometria, interferindo no diagnóstico e qualidade do tratamento endodôntico.

A busca por $100 \%$ de adequação na qualidade radiográfica dos tratamentos endodônticos nunca é desnecessária, mesmo levando em consideração a dificuldade da técnica endodôntica, principalmente, quando executada por acadêmicos dos primeiros semestres de disciplinas clínicas. Para isso, fazse necessário um maior treinamento prático dos alunos, na disciplina de Endodontia Laboratorial, para melhorar sua habilidade manual e diminuir a ocorrência de erros na prática clínica.

CONCLUSÃO 
nesse estudo, pode-se concluir que o perfil dos pacientes, cujos tratamentos endodônticos foram avaliados radiograficamente, foi predominantemente do sexo feminino e faixa etária de 21 a 30 anos. Já o grupo dentário dos elementos mais encontrados para a avaliação radiográfica foi 0 dos incisivos centrais superiores.

Com relação às obturações realizadas, a maioria apresentou conicidade cônica, densidade e limite apical adequados, sugerindo que a metodologia de ensino e aprendizagem oferecidos pela disciplina de Endodontia mostram resultados satisfatórios. Apesar de algum tipo de erro de obturação ser observada em algumas radiografias, a maioria dos tratamentos endodônticos avaliados encontravam-se aceitáveis de acordo com o limite apical e densidade de obturação.

Portanto, algumas sugestões para aumentar o índice de tratamentos aceitáveis e diminuir o número de erros de obturação seriam o aumento da carga horária de aulas práticas de Endodontia laboratorial e estratégias inovadoras mais rígidas, adotadas pelos docentes, de acompanhamento durante a prática clínica. Além disso, novas pesquisas devem ser realizadas nos anos posteriores a fim de comparar os resultados e verificar uma melhor adequação dos itens avaliados.

REFERÊNCIAS

1. Estrela C, Holland R, Estrela CRA, Alencar AHG, Sousa-Neto MD, Pécora JD. Characterization of Successful Root Canal Treatment. Braz Dent J. 2014;25(1):3-11.

2. Zoti M, Hartmann MSM. Avaliação de tratamentos endodônticos realizados por alunos de graduação da Escola de Odontologia da IMED. J Oral Invest. 2016;5(1):4-12.

3. Eskandarloo A, Karkehabadi H, Hoseini Hashemi SZ, Ahmadi M, Hendi SS. Radiographic quality of root canal filling performed by fifth-year students at the Faculty of Dentistry of Hamadan. Iran Endod J. 2017; 12(2):236-41.

4. Fong W, Heidarifar O, Killough S, Lappin MJ, El Karim IA. An audit on technical quality of root fillings performed by undergraduate students. Int Endod J. 2018;51(Suppl 3):e197-203.

5. Ribeiro DM, Réus JC, Felippe WT, PachecoPereira C, Dutra KL, Santos JN et al. Technical quality of root canal treatment performed by undergraduate students using hand instrumentation: a meta-analysis. Int Endod J. 2018;51(3):269-83.

6. Siqueira JF Jr, Rôças IN, Alves FR, Campos LC. Periradicular status related to the quality of coronal restorations and root canal fillings in a
Brazilian population. Oral Surg Oral Med Oral Pathol Oral Radiol Endod. 2005;100(3):369-74.

7. European Society of Endodontology. Quality guidelines for endodontic treatment: consensus report of the European Society of Endodontology. Int Endod J. 2006;39(12):921-30.

8. Souza PO, Tonelli SQ, Lima MSFF, Sá PPA, Almeida CKM, Nunes E et al. Abordagem clínico-radiográfica de dentes tratados endodonticamente: status periapical e qualidade das obturações e restaurações coronárias. RENOME. 2019;8(1):77-83.

9. Mohammad N, Fatah FA, Ghazali N, Muhamad AZ, Hamir N. Endodontic root canal treatment performed by undergraduate dental students: Identification of technical standard and postendodontic restorations. Mal J Fund Appl Sci. 2019;15(4):600-3.

10. Miranda LH, Dantas WCF, Mattar C. Técnicas avançadas de obturação endodôntica. Rev FAIPE. 2017:3(1):46-60.

11. Chueh LH, Chen SC, Lee CM, Hsu YY, Pai SF, Kuo ML, Chen CS, Duh BR, Yang SF, Tung YL, Hsiao CK. Technical quality of root canal treatment in Taiwan. Int Endod J. 2003;36(6):416-22.

12. Soares GT. Qualidade dos tratamentos endodônticos realizados por estudantes de odontologia na clínica de endodontia da UFPB [monografia]. João Pessoa: Universidade Federal da Paraíba; 2010.

13. Kamaura D, Carvalho GL, Antoniazzi JH, LageMarques JLS. Avaliação do desempenho dos alunos de graduação durante a prática da técnica endodôntica. Rev ABENO. 2003; 3(1):33-40.

14. Pontes ALB, Machado FCA, Costa APS, Noro LRA, Araujo ME, Ferreira MAF. Avaliação da qualidade dos tratamentos endodônticos em centros de especialidade da grande Natal. Pesq Bras Odontoped Clin Integr. 2013;13(2):155-60.

15. Balto H, Al Khalifah Sh, Al Mugairin S, Al Deeb M, Al-Madi E. Technical quality of root fillings performed by undergraduate students in Saudi Arabia. Int Endod J. 2010;43(4):292-300.

16. Occhi PGI, Souza AA, Rodrigues V, Tomazinho LF. Avaliação de sucesso e insucesso dos tratamentos endodônticos realizados na clínica odontológica da UNIPAR. Rev UNINGÁ. 2011; 8(2):39-46.

17. Ferreira HLJ, Paula MVQ, Guimarães SMR. Avaliação radiográfica de obturações de canais radiculares. Rev Odonto Ciênc. 2007;22(58): 340-45.

18. Ribeiro DM, Henckel MD, Mello FW, Felippe MCS, Felippe WT. Radiographic analysis the obturation's quality in root canal treatment performed by a South Brazilian sample of undergraduate students. RGO Rev Gaúch Odontol. 2019;67:e20190040. 
19. Gaspar Júnior AA, Pinheiro JT, Couto GBL, Soares RPF, Neves CAF. Avaliação radiográfica dos tratamentos endodônticos encontrados nos estudantes de odontologia no Estado de Pernambuco. Odontol Clín Cient. 2009;8(1):29-34.

20. Freitas RG, Cogo DM, Kopper PMP, Santos RB, Gracca FS. Avaliação da qualidade das obturações endodônticas realizadas por estudantes de graduação. RFOPOA. 2008; 49(3):24-7.

21. Silveira FS, Moraes VR, Rodrigues DC. Avaliação de tratamentos endodônticos em acadêmicos da faculdade de odontologia da universidade de Itaúna/MG. RGO Rev Gaúch Odontol. 2002;50(3):133-36.

22. Giusti EC, Puertas KV, Santos EM, Bussadori SK, Martins MD, Nagatani VS et al. Avaliação radiográfica da qualidade de tratamentos endodônticos realizados por especialistas de um plano de saúde odontológico. ConScientiae Saúde. 2007;6(2):371-75.

23. Er O, Sagsen B, Maden M, Cinar S, Kahraman Y. Radiographic technical quality of root fillings performed by dental students in Turkey. Int Endod J. 2006;39(11):867-72.

24. Costa BE, Lima GM, Tonelli SQ, Nunes E, Silveira FF. Incidence and quality of root canal fillings in undergraduate dental students. RGO Rev Gaúch Odontol. 2019;67:e20190057.

25. Román-Richon S, Faus-Matoses V, AlegreDomingo T, Faus-Llácer VJ. Radiographic technical quality of root canal treatment performed ex vivo by dental students at Valencia University Medical and Dental School, Spain. Med Oral Patol Oral Cir Bucal. 2014;19(1):93-7.

26. Vukadinov T, Blažić L, Kantardžić I, Lainović T. Technical quality of root fillings performed by undergraduate students: a radiographic study. ScientificWorldJournal. 2014;2014:751274.

27. Alves-Silva EG, Souza PXL, Leite NTS, Carvalho RA, Dametto FR, Gomes BPFA et al. Análise da qualidade das radiografias executadas durante os tratamentos endodônticos realizados na graduação da Universidade Potiguar. Arch Health Invest. 2020;9(3):216-21.

\section{CONFLITO DE INTERESSES}

Os autores declaram não haver conflitos de interesse

\section{AUTOR PARA CORRESPONDÊNCIA}

\section{Fábio Victor Dias Silva}

Centro Universitário de João Pessoa (UNIPÊ)

Rodovia BR 230 - Km 22, S/N - Água Fria

58053-000 João Pessoa - PB, Brasil

Email: fabio_vct@hotmail.com

Submetido em 20/01/2021

Aceito em 08/03/2021 